\title{
Bernard and Joan Marshall Awards at the Autumn Meeting of the British Society for Cardiovascular Research 2017.
}

Carolyn A Carr, Michael S Dodd, Lisa C Heather

Department of Physiology, Anatomy \& Genetics, Sherrington Building, Parks Road, Oxford OX1 3PT

The Bernard and Joan Marshall Awards of the British Society for Cardiovascular Research (BSCR) were introduced in 2010. The Society was founded in 1973 by Professor David Hearse of the Rayne Institute, St Thomas' Hospital, London, and was initially called the Cardiac Muscle Research Group. At that time, the British Cardiac Society did not admit basic scientists and Professor Hearse felt that Britain needed a forum in which researchers could exchange ideas and present their results. The BSCR continues with its main aim being to provide a forum for scientists to discuss their research and, in particular, to provide a platform for students and early post-docs to present their work. To facilitate this, the society organises two main meetings a year. The Spring Meeting forms part of the annual conference of the British Cardiovascular Society (as the British Cardiac Society now is) and is organised with the British Atherosclerosis Society to provide the basic science wing of that conference. The Autumn Meeting is more of a themed meeting and includes a session dedicated to the Bernard and Joan Marshall Awards.

Bernard and Joan Marshall were the uncle and aunt of Professor David Hearse and they followed his work with great interest. Bernard Marshall served in the Royal Navy during the Second World War and then worked for the Ministry of Agriculture, Fisheries and Food for many years, rising to a senior level by his retirement. Bernard set up the Tenant Farmer's Association, becoming its first Director General, and was an Honorary Research Fellow at the Centre for Agricultural Strategy at the University of Reading. In this role, he organised various projects and national conferences and clearly understood the value of conferences for the exchange of ideas. Bernard and Joan were impressed with David's work to encourage young scientists and they very generously bequeathed a sum of money to the BSCR to encourage, recognise and support the work of young investigators, and to facilitate an inspirational keynote lecture at the annual meeting of the Society.

The Bernard and Joan Marshall Awards currently recognise excellence at three stages of an academic career. The Young Investigator Award is a competitive award open to students and post-docs who are within 3 years of the award of their PhD, DPhil or MD. Applicants submit a full manuscript that is in preparation, or submitted, or is a paper published within the preceding 12 months. The top three applicants are invited to present their work at the Autumn Meeting, to 
compete for the Bernard and Joan Marshall Young Investigator Prize. In 2017, the three candidates selected to present were Dr Tom Parks from the University of Oxford who discussed his paper entitled 'Association between a common immunoglobulin heavy chain allele and rheumatic heart disease risk in Oceania'1; Dr Eylem Levelt from the University of Leicester who presented her work on 'Cardiac energetics, oxygenation, and perfusion during increased workload in patients with type two diabetes mellitus'2; and Dr Johannes Bargehr from the University of Cambridge (figure 1) who presented his data on 'Human embryonic stem cellderived epicardial cells augment cardiomyocyte-driven heart regeneration' (under review). All three talks were stimulating and informative and resulted in lively question-and-answer sessions. After much consultation, the judges awarded the Bernard and Joan Marshall Young Investigator Prize to Dr Bargehr for his elegant work to investigate the beneficial effect of a combined graft of embryonic stem cell (ESC)-derived epicardial cells and cardiomyocytes. Starting from in vitro data showing that ESC-derived epicardial cells enhance contractility of 3D engineered heart tissue constructs, this work progressed to in vivo studies where administration of combined grafts had better functional benefit in the infarcted rat heart than either cell type alone.

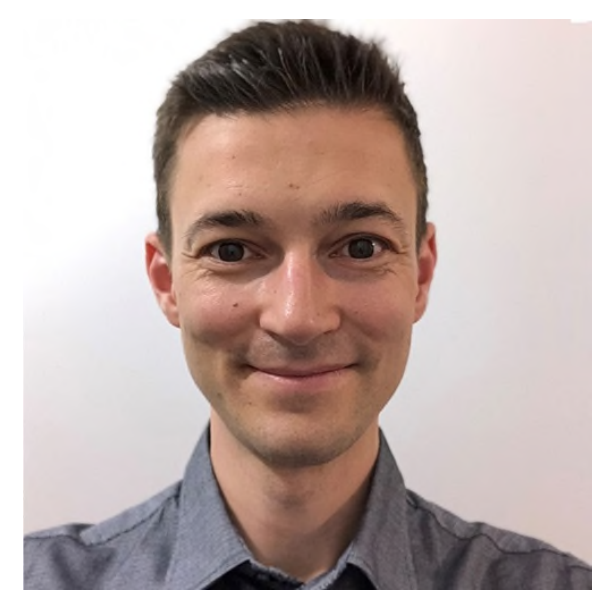

Figure 1: Dr Johannes Bargehr.

The Research Excellence Award is also a competitive award, for established independent investigators who have received their higher degree in the preceding 15 years. Applicants are invited to submit a summary of their research achievements and a manuscript in preparation or recently published. At the 2017 Autumn Meeting, the Research Excellence Award was presented to Dr Stephen Wheatcroft from the University of Leeds (figure 2). His research focuses on understanding how insulin resistance causes adverse vascular remodelling and in identifying how the family of the insulin-like growth factor binding proteins (IGFBPs) may offer an approach to ameliorating the adverse effects on the vascular endothelium. In his lecture, he discussed his work showing that overexpression of IGFBP1 in mice on a high-fat diet reduced 
blood pressure, improved whole-body insulin sensitivity and increased vascular nitric oxide generation. Similarly, in insulin-resistant mice, IGFBP1 overexpression reduced blood pressure and increased insulin-stimulated nitric oxide generation. ${ }^{3}$ Furthermore, he showed that IGFBP1 confers protection against atherosclerosis and can ameliorate the effects of insulin resistance on endothelial repair processes. ${ }^{3}$ In work published in a recent paper, ${ }^{4}$ he described how IGFBP1 improves glucose regulation and insulin sensitivity by increasing insulin-stimulated activation of the Akt pathway via signalling through the RGD domain, thereby opening a potential new way to achieve insulin sensitisation in patients.

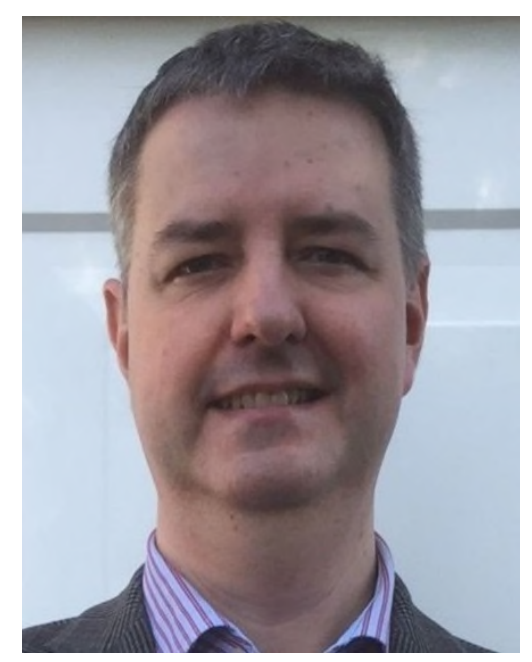

Figure 2: Dr Stephen Wheatcroft

The Distinguished Investigator Award is given to someone who has made a substantial contribution to cardiovascular research, as selected by the BSCR committee and the organisers of the Autumn Meeting. The Distinguished Investigator receives an honorarium and presents the Keynote lecture at the meeting. In 2017, this award was presented to Professor Rong Tian, Director of the Mitochondria and Metabolism Centre at the University of Washington (figure 3). Her research focuses on the molecular mechanisms regulating cell metabolism and energetics, and in particular on the role of mitochondrial metabolism in cardiovascular diseases. Having identified specific regulators in the key pathways of cardiac energy metabolism in mice, she uses multinuclear NMR spectroscopy to explore the physiological and biochemical responses in vivo during the development of heart failure. In her lecture, she began by describing how she has shown that cardiac-specific overexpression of the glucose transporter GLUT1 prevents the increase in fatty oxidation induced in the hearts of wild-type mice on a high-fat diet, but that this results in increased oxidative stress in the heart and contractile dysfunction. ${ }^{5}$ The remodelling of the metabolic network included an increase in acetyl-CoA carboxylase (ACC), and she went on to show that cardiac-specific deletion of ACC2 in the mouse attenuated pressure-overload hypertrophy, with increased fatty acid oxidation and improved function and energetics. ${ }^{6}$ Returning to the GLUT1-overexpressing mouse heart, she showed that these hearts also 
maintained cardiac energetics and function under pressure overload, but that in this case the hearts still showed hypertrophy. ${ }^{7}$ Rong Tian then described recent work that investigated the effect of branched chain amino acid (BCAA) catabolism on cardiac energy metabolism and function using mice deficient in the serine-threonine protein phosphatase $\mathrm{PP} 2 \mathrm{Cm} .{ }^{8}$ Using a combination of phosphorous-31 and carbon-13 nuclear magnetic resonance (NMR) spectroscopy of isolated perfused mouse hearts, she showed that contractile function was similar in wild-type and PP2Cm-deficient hearts, and that the phosphocreatine-to-ATP ratio, an estimate of myocardial energetics, was also comparable between the two groups. However, there was a switch towards increased fatty acid oxidation in the PP2Cm-deficient hearts resulting from a chronic accumulation of BCAA, which inhibited the pyruvate dehydrogenase complex activity. The PP2Cm-deficient mouse hearts were found to be vulnerable to ischaemia/reperfusion injury, but this could be rescued by increasing BCAA catabolism or by overexpressing GLUT1 and thereby normalising glucose use. ${ }^{8}$ Finally, she described recent work showing that elevated glucose levels decreased expression of Kruppel-like factor 15 (KLF15) and that this in turn downregulated BCAA degradation. ${ }^{9}$ She concluded by saying that this effect acted as a checkpoint to promote growth when other hypertrophic stimuli were present.

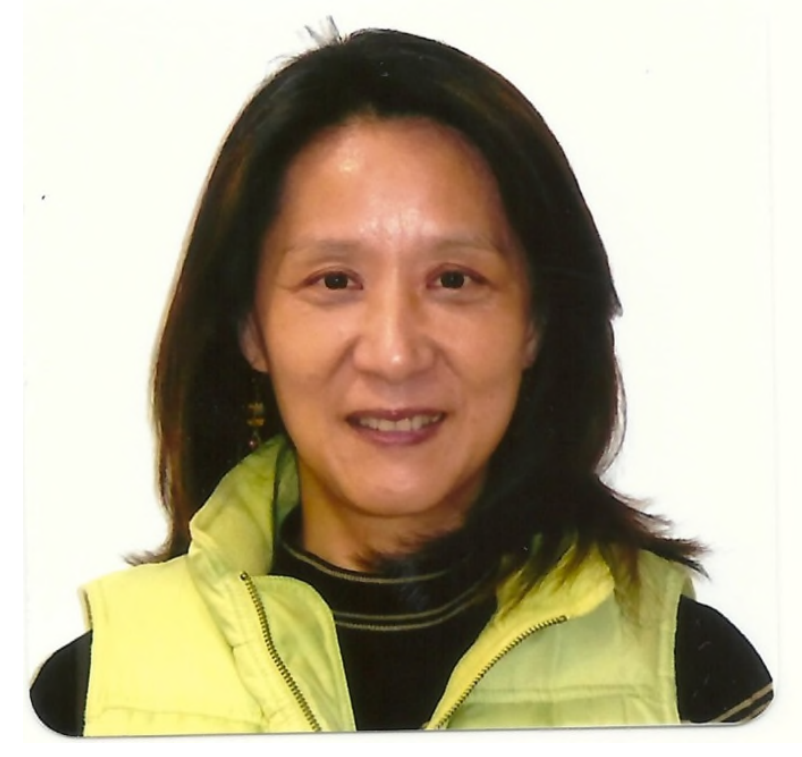

Figure 3: Professor Rong Tian.

Thus, the Bernard and Joan Marshall Awards have again enabled the BSCR to showcase some of the best young investigators in the society and to invite leaders in the field to give inspirational presentations of their work. 


\section{References}

1 Parks T, Mirabel MM, Kado J, et al. Association between a common immunoglobulin heavy chain allele and rheumatic heart disease risk in Oceania. Nat Commun 2017;8. doi:10.1038/ncomms14946

2 Levelt E, Rodgers CT, Clarke WT, et al. Cardiac energetics, oxygenation, and perfusion during increased workload in patients with type 2 diabetes mellitus. Eur Heart J 2016;37:3461-9. doi:10.1093/eurheartj/ehv442

3 Abbas a., Imrie H, Viswambharan $\mathrm{H}$, et al. The Insulin-Like Growth Factor-1 Receptor Is a Negative Regulator of Nitric Oxide Bioavailability and Insulin Sensitivity in the Endothelium. Diabetes 2011;60:2169-78. doi:10.2337/db11-0197

4 Haywood NJ, Cordell PA, Tang KY, et al. Insulin-Like Growth Factor Binding Protein 1 Could Improve Glucose Regulation and Insulin Sensitivity Through Its RGD Domain. Diabetes 2017;66:287-99. doi:10.2337/db16-0997

5 Yan J, Young ME, Cui L, et al. Increased glucose uptake and oxidation in mouse hearts prevent high fatty acid oxidation but cause cardiac dysfunction in diet-induced obesity. Circulation 2009;119:2818-28. doi:10.1161/CIRCULATIONAHA.108.832915

6 Kolwicz SC, Olson DP, Marney LC, et al. Cardiac-specific deletion of acetyl CoA carboxylase 2 prevents metabolic remodeling during pressure-overload hypertrophy. Circ Res 2012;111:728-38. doi:10.1161/CIRCRESAHA.112.268128

7 Liao R, Jain M, Cui L, et al. Cardiac-specific overexpression of GLUT1 prevents the development of heart failure attributable to pressure overload in mice. Circulation 2002;106:2125-31. doi:10.1161/01.CIR.0000034049.61181.F3

8 Li T, Zhang Z, Kolwicz SC, et al. Defective Branched-Chain Amino Acid Catabolism Disrupts Glucose Metabolism and Sensitizes the Heart to Ischemia-Reperfusion Injury. Cell Metab 2017;25:374-85. doi:10.1016/j.cmet.2016.11.005

9 Shao D, Villet O, Zhang Z, et al. Glucose Promotes Cell Growth by Suppressing Branchedchain Amino Acid Degradation. JMCC 2017;112:156. 